\title{
Magnetic behaviour and magnetocaloric effect of neodymium-based amorphous alloy
}

\author{
S. Gorsse ${ }^{\text {a) }}$, G. Orveillon, B. Chevalier \\ Institut de Chimie de la Matière Condensée de Bordeaux, \\ ICMCB, CNRS, Université Bordeaux 1 \\ 87 Av. Dr A.Schweitzer, 33608 PESSAC Cedex, France
}

\begin{abstract}
The $\mathrm{Nd}_{49} \mathrm{Al}_{13} \mathrm{Ni}_{38}$ amorphous alloy has been prepared by melt-spinning in the form of ribbons. Its magnetic properties have been investigated via superconducting quantum interference device magnetometry and its magnetic phase diagram was established. Hysteresis and temperature-dependent magnetization measurements show the occurrence of a re-entrant spin glass behaviour on cooling. With increasing applied field, the spin-freezing temperature decreases and disappears at very high field, and the Curie temperature increases, broadening the temperature range of the ferromagnetic state. The resulting magnetocaloric effect (MCE) was evaluated and compared to others interesting magnetic refrigerant materials.
\end{abstract}

a) Corresponding author - gorsse@icmcb-bordeaux.cnrs.fr 


\section{Introduction}

Magnetocaloric effect (MCE) consists in temperature change of magnetic materials upon the application of a magnetic field. MCE attracts very much attention due to its potential application in environmentally-friendly and high energy efficiency magnetic refrigeration [1]. The rejection and absorption of heat is accomplished by adiabatic temperature change upon magnetization and demagnetization of the magnetic refrigerant. Families of magnetic materials which exhibit large MCE value resulting on a large field-induced entropy change include rare-earth (RE) intermetallics $\left(\mathrm{REM}_{2}\right.$ where $\mathrm{M}=\mathrm{Al}$, Co and $\left.\mathrm{Ni}\right)$ and their hydrides, and manganites $\left(\mathrm{RE}_{1-\mathrm{x}} \mathrm{M}_{\mathrm{x}} \mathrm{MnO}_{3}\right.$ where $\mathrm{M}=\mathrm{Ca}$, $\mathrm{Sr}$ and $\left.\mathrm{Ba}\right)$ [2]. Recently, magnetocaloric effect of Fe- [3, 4, 5, 6, 7, 8, 9, 10, 11, 12,], Co- [13], Gd-based [14, 15, 16, 17] and Pd- [18] amorphous alloys have been examined because they display interesting properties for a suitable magnetic refrigerants, e.g. a high electric resistivity that decreases eddy current losses, a tuneable ordering temperature, high corrosion resistance and outstanding mechanical properties.

Amorphous alloys can present a large variety of magnetic properties such as paramagnetism, superparamagnetism, superferromagnetism, and spin glass, depending of their method of preparation (e.g. melt-spinning at different cooling rates, copper mold casting, post annealing) and composition. The disordered structure in amorphous ferromagnets gives rise to random isotropic exchange and random anisotropy resulting of local easy axes whose varies randomly in space, instead of having a global anisotropy along crystallographic axes. According to the random anisotropy model [19], the magnetic behaviour can dramatically be affected by the random anisotropy; depending on its strength disordered phases can exhibit soft or hard magnetic behaviour [20]. Having a magnetic hysteresis reduced to nullity is 
particularly interesting for magnetic refrigeration in which energy losses during magnetization/demagnetization cycles must be minimized.

The strength of the random anisotropy and, in consequence, the magnetic properties and MCE of amorphous alloys can be tailored via the choice of the RE solvent element. In RE compounds the magnetic anisotropy results of the spin orbit coupling and depends on the $\mathrm{J}$ state of the RE ion. In this perspective, we investigate the properties of a series of RE-Al-Ni amorphous alloys with $\mathrm{RE}=\mathrm{La}, \mathrm{Ce}, \mathrm{Nd}, \mathrm{Sm}, \mathrm{Gd}$ and Dy because of their different J states and magnetic moments. In this paper, we present the magnetic properties and the MCE properties of $\mathrm{Nd}_{49} \mathrm{Al}_{13} \mathrm{Ni}_{38}$ amorphous alloy.

\section{Experiment}

The alloy composition, $\mathrm{Nd}_{49} \mathrm{Al}_{13} \mathrm{Ni}_{38}$, was designed based on topologic and thermodynamic criteria [21] to ensure a good glass forming ability. The master alloy was prepared by melting precisely weighed amounts of high purity elements $\mathrm{Nd}$ (99.9\%), $\mathrm{Al}$ (99.999\%) and Ni (99.995\%), in a levitation-furnace. Melting was carried out in a watercooled copper crucible under a purified argon atmosphere and performed several times to achieve good homogeneity. The amorphous alloy is produced in the form of ribbons by single-roller melt-spinning technique with a circumferential speed of $40 \mathrm{~m} / \mathrm{s}$ in a purified argon atmosphere.

The amorphous state of the melt-spun ribbon was verified by X-ray diffraction (XRD) and differential scanning calorimetry (DSC).

In order to gain a detailed picture of the magnetic properties of $\mathrm{Nd}_{49} \mathrm{Al}_{13} \mathrm{Ni}_{38}$ melt-spun ribbons, we have measured the temperature and field dependence of dc magnetic susceptibilities. These measurements were performed using a superconducting quantum 
interface device (SQUID) magnetometer. Heat capacity was also measured with a Physical Properties Measurement System (PPMS) from Quantum Design in temperature range of 1.8$300 \mathrm{~K}$.

\section{Results and discussion}

The XRD pattern of the $\mathrm{Nd}_{49} \mathrm{Al}_{13} \mathrm{Ni}_{38}$ melt-spun ribbons is shown in Figure 1. Only two broad diffraction peaks are observed which indicates a fully amorphous structure. The occurrence of a glass transition at $524 \mathrm{~K}$ further confirms the amorphous nature of the sample (Fig. 2).

Plots of the ratio $\mathrm{H} / \mathrm{M}(\mathrm{H}$, the applied field, and $\mathrm{M}$, the magnetization) as a function of the temperature (Fig. 3), and at various applied field $\left(\mu_{0} \mathrm{H}\right)$ ranging from $0.005 \mathrm{~T}$ to $1 \mathrm{~T}$, shows that the $\mathrm{Nd}_{49} \mathrm{Al}_{13} \mathrm{Ni}_{38}$ amorphous alloy obeys to a Curie-Weiss law at high temperature with a paramagnetic Curie temperature $\theta_{\mathrm{p}} \approx 15 \mathrm{~K}$ and an effective moment $\mu_{\mathrm{eff}}$ of $3.46 \mu_{\mathrm{B}} / \mathrm{Nd}$. This is very close to the expected value of $3.62 \mu_{\mathrm{B}}$ for $\mathrm{Nd}^{3+}$ ions which suggests, together with the positive value of $\theta_{\mathrm{p}}$, a ferromagnetic (FM) spin coupling between RE ions. With decreasing temperature, the ratio $\mathrm{H} / \mathrm{M}$ starts to deviate from the Curie-Weiss behaviour at the temperature $T_{d}<30 \mathrm{~K}$ depending on the applied field. This deviation reflects the establishment of short range exchange interactions giving rise to small correlated volume, i.e. magnetic clusters with a correlation length that increases as the temperature decreases below $\mathrm{T}_{\mathrm{d}}$

Figure 4 presents the $M(H)$ magnetic loops at various temperatures after zero field cooling (ZFC) and the inset shows the first magnetization curves at various temperatures. At 
$270 \mathrm{~K}$, the magnetism can be described with paramagnetism (PM). At 30 and $15 \mathrm{~K}$, the Sshape of the magnetisation loop with no coercivity is typical of superparamagnetism (SPM) this behaviour is confirmed below from the analysis of the $\mathrm{M}$ vs $\mathrm{H} / \mathrm{T}$ data using the Langevin function. At $9 \mathrm{~K}$, the behaviour is characteristic of ferromagnetism (FM) with an increasing coercivity as the temperature decreases. The $\mathrm{Nd}_{49} \mathrm{Al}_{13} \mathrm{Ni}_{38}$ melt-spun ribbon is a soft magnet at $9 \mathrm{~K}$ and a harder magnet at $1.8 \mathrm{~K}$ and $3 \mathrm{~K}$ with coercivity of 0.3025 and $0.1100 \mathrm{~T}$, and remanence of 17.3 and $11.7 \mathrm{Am}^{2} / \mathrm{kg}$, respectively. In the inset of Figure 4, one can also see a decrease of the permittivity as the temperature is lowered. Higher coercivity and lower permittivity at low $\mathrm{T}$ reveal the presence of a magnetic anisotropy due to the $\mathrm{L}$ state of $\mathrm{Nd}^{3+}$. Even at 4.6 T, complete saturation is never achieved which can be attributed to the progressive flipping of spins to a ferromagnetic alignment and suggests the presence of antiferromagnetic interactions between $\mathrm{Nd}^{3+}$ ions. In a disordered system containing $\mathrm{RE}$, the variation of local environments and distances can cause the intensity and sign of the RKKY exchange interactions to fluctuate in space, and, as a consequence, the spins in a given magnetic cluster are not necessary all ferromagnetically aligned in the same direction.

In Figure 5, the zero-field-cooled (ZFC) and field-cooled (FC) magnetization curves $M(T)$ of the amorphous alloy show a paramagnetic-ferromagnetic phase transition at $\mathrm{T}_{\mathrm{C}}=11$ $16 \mathrm{~K}$, and a thermomagnetic irreversibility between ZFC and FC conditions at $\mathrm{T}_{\text {irr }}=5-9 \mathrm{~K}$. This difference at low $\mathrm{T}$ between $\mathrm{ZFC}$ and FC curves is attributed to the local random magnetic anisotropy. The irreversibility is below approximately $10 \mathrm{~K}$, depending of the applied field. Above this bifurcation, ZFC and FC curves superimpose perfectly while below, the ZFC branch decreases rapidly and the FC branch increases to a plateau. The maxima of the ZFC curve decreases to lower $\mathrm{T}$ with increasing field from 0.005 to $0.1 \mathrm{~T}$, and matches with the temperature of irreversibility, $\mathrm{T}_{\text {irr. }}$. At higher field $\left(\mu_{0} \mathrm{H}=1 \mathrm{~T}\right), \mathrm{ZFC}$ and FC curves 
superimpose in the whole temperature range from $1.8 \mathrm{~K}$ to $300 \mathrm{~K}$ and encounter a plateau below $10 \mathrm{~K}$. Possible origins of this overall behaviour include surface-spin-glass freezing, cooperative freezing of interacting magnetic clusters (superferromagnetism), re-entrant spin glass transition or superparamagnetism blocking. Xu et al. [22] observed for an amorphous alloy with similar composition (i.e. $\mathrm{Nd}_{53} \mathrm{Al}_{15} \mathrm{Ni}_{32}$ ) and prepared in the same way, that the $\mathrm{ZFC}$ peak around $10 \mathrm{~K}$ moves to higher temperature with increasing frequency in ac susceptibility. This suggests a spin glass with re-entry [23], i.e. the spin glass phase develops from a FM state and it re-enters the frozen disordered phase out of another paramagnetic disordered phase (PM). More details of the temperature dependence of the magnetisation are given by the derivative of $M$ (inset of Fig. 5). The $d M / d T$ vs $T$ curves were plotted for each applied field $H$. To facilitate the lecture, logarithm scale is used for the temperature axis. The ordering temperature $T_{c}$ for $P M$ to $F M$ transition and the freezing temperature $T_{f}$ for $F M$ to $S G$ transition were determined at the kinks (Table 1). The bifurcation of ZFC and FC $d M / d T$ curves denotes the progressive freezing of spins or group of spins (clusters) below $\mathrm{T}_{\text {irr }}$. Figure 6 shows that the field dependence of $\mathrm{T}_{\mathrm{f}}$ and $\mathrm{T}_{\text {irr }}$ follow the Almeida-Thouless (AT) line with a $\mathrm{H}^{1 / 2}$ law which has also been observed in re-entrant SG systems [24] and predicted by the Sherrington-Kirkpatrick model of SG [25].

The magnetic ordering transition observed on the $\mathrm{M}(\mathrm{T})$ curves is also visible in the temperature dependence of the heat capacity, $C_{p}(T)$, shown in Figure 7. Only one small anomaly around $10 \mathrm{~K}$ is observed, which is characteristic of a second order phase transition. This anomaly shifts to higher temperature with increasing the applied field, from $10 \mathrm{~K}$ for $\mu_{0} \mathrm{H}=0 \mathrm{~T}$ to $12 \mathrm{~K}$ for $\mu_{0} \mathrm{H}=1 \mathrm{~T}$ (inset of Fig. 7). These values are close to $\mathrm{T}_{\mathrm{c}}$ obtained above from magnetisation curves (Fig. 5 and Table 1). 
From the above results we can draw the following picture to explain the overall magnetic behaviour of the $\mathrm{Nd}_{49} \mathrm{Al}_{13} \mathrm{Ni}_{38}$ amorphous alloy. At high temperature, there is one PM phase made of weakly interacting $\mathrm{Nd}^{3+}$; the thermal dependence of the magnetization is well reproduced by the Brillouin function (Fig. 8). Between $T_{d}$ and $T_{C}$, the competition between ordering due to exchange interaction and the disordering effect of temperature gives rise to the formation of ordered magnetic clusters embedded into a PM matrix, when short range exchange for a group of spins becomes higher than $\mathrm{kT}$. A quantitative analysis using the Langevin function confirms this description and shows an increase of the number of clusters as the temperature is lowered (Fig. 8). On cooling, these correlated volumes grow resulting of an increase of the correlation length up to the infinite magnetic cluster with wandering axes. Below $\mathrm{T}_{\text {irr }}$, the hysteretic behaviour results of the progressive broken up of the magnetically ordered phase by the local anisotropy into frozen and randomly oriented clusters. The presence of coercivity indicates that the anisotropic clusters formed below $T_{\text {irr }}$ have a size comparable to the exchange coupling correlation length. As the temperature is kept decreasing less and less spin can overcome the local anisotropy, and below $\mathrm{T}_{\mathrm{f}}$ spins are frozen along their local anisotropy axes.

The H-T phase diagram (Fig. 9) consists of two disordered phases (PM and SG) and ordered magnetic clusters, separated by binary regions in which magnetically correlated volumes are formed into one of the disordered phase.

The resulting magnetocaloric response of the $\mathrm{Nd}_{49} \mathrm{Al}_{13} \mathrm{Ni}_{38}$ amorphous alloy has been derived from Maxwell relation by integrating over the magnetic field:

$\left(\frac{\partial S}{\partial M}\right)_{T}=\left(\frac{\partial M}{\partial T}\right)_{H} \quad$ where $S$ represents the magnetic entropy.

The magnetic entropy change, $\Delta S_{M}$, produced by the variation of a magnetic field from 0 to $\mathrm{H}_{\max }$ is given by 
$\Delta S_{M}(T, H)=\int_{0}^{H_{\max }}\left(\frac{\partial M}{\partial T}\right)_{H} d H$

and the adiabatic temperature change, $\Delta T_{a d}$, linked to MCE can be expressed as $\Delta T_{a d}=-\left(T / C_{0}(T)\right) \Delta S_{M}$.

Figure 10 shows the temperature dependence of the magnetic entropy change, $\Delta S_{M}(T)$, corresponding to an applied field $\mathrm{H}$ of $1 \mathrm{~T}$. The behaviour shows several remarkable features. There are two peaks corresponding to the SG to FM transition at $T_{f}$ and the FM to PM transition at $T_{c}$, with a positive and negative value, respectively. The peak entropy change at $\mathrm{T}_{\mathrm{c}}$ is $\Delta S_{M}=-1.6 \mathrm{~J} \cdot \mathrm{kg}^{-1} \cdot \mathrm{K}^{-1}$, corresponding to the adiabatic temperature change $\Delta T_{a d}=5.4 \mathrm{~K}$, which compare well with other known MCE materials (Table 2). The FM to PM transition in the $\mathrm{Nd}_{48} \mathrm{Al}_{13} \mathrm{Ni}_{38}$ amorphous alloy produces a broad peak in the magnetic entropy change (Fig. 10). This characteristic, resulting of the disordered structure of the material, is interesting since it enhances the refrigerant capacity [26]. $\mathrm{T}_{\mathrm{c}}$ is lowered due to the disorder effect that significantly reduces the electron mean free path and in consequence decreases the RKKY coupling at smaller length scale.

\section{Conclusion}

The magnetic behaviour of the $\mathrm{Nd}_{49} \mathrm{Al}_{13} \mathrm{Ni}_{38}$ amorphous alloy goes from paramagnetic, ferromagnetic to spin-glass upon cooling. It is a soft magnet at $9 \mathrm{~K}$ and becomes a hard magnet with higher coercivity at lower temperature. This overall behaviour originates from the formation of magnetic clusters resulting on the competition between the development of short-range spatial magnetic ordering and the disordering effect of the 
random magnetic anisotropy. Given its $\mathrm{H}-\mathrm{T}$ magnetic phase diagram, exhibiting a large panel of magnetic phases (PM, FM, and SG), the $\mathrm{Nd}_{49} \mathrm{Al}_{13} \mathrm{Ni}_{38}$ amorphous alloy provides a good prototype to compare the effect of the strength of the random anisotropy since other choices for the RE element should give rise to modifications of the H-T diagram with enlargement of specific phase fields and reduction of others. In term of MCE, $\Delta S_{m}$ of $\mathrm{Nd}_{49} \mathrm{Al}_{13} \mathrm{Ni}_{38}$ compares well with others amorphous materials. It appears that, in addition of heat treatments that can be performed on the amorphous to produce nanocomposite structures, tuning the strength of the random anisotropy could be another degree of freedom allowing to tailor the magnetic properties and MCE of amorphous alloys.

\section{Acknowledgements}

Financial support from the European Office of Aerospace Research and Development through Research Task FA8655-07-M-4003 is greatly appreciated. SG wishes to thank O. Senkov for DSC experiments. 


\section{References}

$1 \quad$ J. Glanz, Science 279, 2045 (1998).

2 K.A. Gscheidner, V.K. Pechersky, and A.O. Tsokol, Rep. Prog. Phys. 68, 1479 (2005).

3 D. Wang, K. Peng, B. Gu, Z. Han, S. Tang, W. Qin, and Y. DU, J. Alloys Compd. 358, 312 (2003).

$4 \quad$ S. Atalay, H. Gencer, and V.S. Kolat, J. Non-Cryst. Solids 351, 2373 (2005).

5 S.G. Min, K.S. Kim, S.C. Yu, H.S. Suh, and S.W. Lee, J. Appl. Phys. 97, 10M310 (2005).

6 V. Fransco, J.S. Blazquez, C.F. Conde, and A. Conde, Appl. Phys. Lett. 88, 042505 (2006).

$7 \quad$ F. Johnson and R.D. Shull, J. Appl. Phys. 99, 08 K909 (2006).

8 V. Franco, J.S. Blazquez, and A. Conde, J. Appl. Phys. 100, 064307 (2006).

9 V. Franco, J.M. Borrego, A. Conde, and S. Roth, Appl. Phys. Lett. 88, 132509 (2006).

10 V. Franco, J.M. Borrego, C.F. Conde, A. Conde, M. Stoica, and S. Roth, J. Appl. Phys. 100, 083903 (2006).

11 V. Franco, J.S. Blazquez, M. Millian, J.M. Borrego, C.F. Conde, and A. Conde, J. Appl. Phys. 101, 09C503 (2007).

12 S.G. Min, K.S. Kim, S.C. Yu, and K.W. Lee, Mat. Sci. Eng. A 449-451, 423 (2007).

13 P. Didukh and A. Slawska-Waniewska, J. Magn. Magn. Mater. 254/255, 407 (2003).

14 M. Foldeaki, R. Chachine, B.R. Gopal, T.K. Bose, X.Y. Liu, and J.A. Barclay, J. Appl. Phys. 83, 2727 (1998).

15 Q. Luo, D.Q. Zhao, M.X. Pan, and W.H. Wang, App. Phys. Lett. 89, 081914 (2006).

16 L. Si, J. Ding, Y. Li, B. Yao, and H. Tan, Appl. Phys. A 75, 535 (2002). 
17 B. Chevalier, J.-L. Bobet, J. Sanchez Marcos, J. Rodriguez Fernandez, and J.C.

Gomez Sal, Applied Physics A, 80, 601 (2005).

18 T.D. Shen, R.B. Schwarz, J.Y. Coultier, and J.D. Thompson, J. Appl. Phys. 91, 5240 (2002).

19 R. Harris, M. Plisschke, and M.J. Zuckermann, Phys. Rev. Lett. 31, 160 (1973).

20 R. Alben, J.J. Becker, M.C. Chi, J. Appl. Phys. 49, 1653 (1978).

21 G. Orveillon, O.N. Senkov, J.-L Soubeyroux, B. Chevalier, and S. Gorsse, Adv. Eng. Mater. 9, 483 (2007).

22 F. Xu, X. Wu, Y. Du, C. Cui, and G. Chen, J. Appl. Phys. 99, 08B524 (2006).

23 J. A. Mydosh, Spin Glasses (Taylor and Francis, London, 1993).

24 Y.T. Wang, H. Y. Bai, M. X. Pan, D. Q. Zhao, and W. H. Wang, Phys. Rev. B 74, 064422 (2006).

25 J. R. L. de Almeida and D. J. Thouless, J. Phys. A 11, 983 (1978).

26 M. E. Wood and W. H. Potter, Cryogenics 25, 667 (1985). 


\section{Tables}

Table 1: Freezing temperature $\left(\mathrm{T}_{\mathrm{f}}\right)$, temperature of irreversibility $\left(\mathrm{T}_{\text {irr }}\right)$, ferromagnetic transition $\left(\mathrm{T}_{\mathrm{C}}\right)$ and deviation from the Curie-Weiss law $\left(\mathrm{T}_{\mathrm{d}}\right)$ obtained at various applied fields for the $\mathrm{Nd}_{49} \mathrm{Al}_{13} \mathrm{Ni}_{38}$ amorphous alloy.

\begin{tabular}{lcccc}
\hline$\mu_{0} . \mathrm{H}(\mathrm{T})$ & $\mathrm{T}_{\mathrm{f}}(\mathrm{K})$ & $\mathrm{T}_{\text {irr }}(\mathrm{K})$ & $\mathrm{T}_{\mathrm{c}}(\mathrm{K})$ & $\mathrm{T}_{\mathrm{d}}(\mathrm{K})$ \\
\hline & & & & \\
0.005 & 8.7 & 9.5 & 11.5 & 20.0 \\
0.015 & 7.1 & 8.7 & 12.0 & 20.0 \\
0.025 & 6.0 & 7.8 & 12.0 & 20.0 \\
0.1 & 3.6 & 5.5 & 13.2 & 21.5 \\
1 & - & - & 15.8 & 26.0 \\
\hline
\end{tabular}

Table 2: Magnetic entropy change upon applying a field $\mathrm{H}$ at various materials and related parameters.

\begin{tabular}{|c|c|c|c|c|c|}
\hline $\begin{array}{l}\text { Amorphous } \\
\text { alloys }\end{array}$ & $\mathrm{T}_{\mathrm{C}}(\mathrm{K})$ & $\Delta \mathrm{H}(\mathrm{T})$ & $\begin{array}{c}-\Delta \mathrm{S}_{\mathrm{M}} \\
\left(\mathrm{J} \mathrm{kg}^{-1} \mathrm{~K}^{-1}\right)\end{array}$ & $\begin{array}{c}\Delta \mathrm{T}_{\mathrm{ad}} \\
(\mathrm{K})\end{array}$ & References \\
\hline $\mathrm{Nd}_{49} \mathrm{Al}_{13} \mathrm{Ni}_{38}$ & 15.8 & 1 & 1.6 & 5.4 & Present work \\
\hline Fe-based & $125-490$ & 1 & $0.2-1.4$ & - & {$[3-12]$} \\
\hline Pd-based & 94 & 5 & 0.6 & & [18] \\
\hline RE-based & $30-90$ & 5 & $9-12$ & & {$[14-17]$} \\
\hline
\end{tabular}




\section{Figures captions}

Figure 1: DRX pattern of the melt-spun ribbon of $\mathrm{Nd}_{49} \mathrm{Al}_{13} \mathrm{Ni}_{38}$.

Figure 2: Heat flow vs. temperature curves of the $\mathrm{Nd}_{49} \mathrm{Al}_{13} \mathrm{Ni}_{38}$ amorphous alloy at the heating rate of $40 \mathrm{~K} / \mathrm{min}$. The exothermic reactions are up.

Figure 3: $\mathrm{H} / \mathrm{M}$ ratio plots of the $\mathrm{Nd}_{49} \mathrm{Al}_{13} \mathrm{Ni}_{38}$ amorphous alloy at various applied field $\left(\mu_{0} \mathrm{H}=\right.$ 0.1 and $1 \mathrm{~T})$. The line represents the Curie-Weiss law at high temperatures $\left(\mathrm{R}^{2}=0.999\right)$.

Figure 4: Magnetic hysteresis loops $M(H)$ of the $\mathrm{Nd}_{49} \mathrm{Al}_{13} \mathrm{Ni}_{38}$ amorphous alloy at various temperatures ( $\mathrm{T}=270,30,15,9,3$ and $1.8 \mathrm{~K})$. The inset shows the first magnetisation curves at the same temperatures.

Figure 5: Zero-Field-Cooled (ZFC - open symbols) and Field-Cooled (FC - full symbols) magnetization curves $M(T)$ of the $\mathrm{Nd}_{49} \mathrm{Al}_{13} \mathrm{Ni}_{38}$ amorphous alloy at various applied field $\left(\mu_{0} \mathrm{H}\right.$ $=0.005,0.015,0.025,0.1$, and $1 \mathrm{~T})$. The inset represents the ZFC-FC derivates of magnetization $d M / d T$ vs $T$ for each applied field $H$. A log-scale is used for $T$.

Figure 6: Field dependence of freezing temperature $\left(T_{f}\right)$ and temperature of irreversibility ( $\mathrm{T}_{\text {irr }}$ ). The lines represent the Almeida-Thouless $\mathrm{H}^{1 / 2}$ dependence law.

Figure 7: Specific heat capacity at constant pressure of the $\mathrm{Nd}_{49} \mathrm{Al}_{13} \mathrm{Ni}_{38}$ amorphous alloy without (open circles) and with (full triangles) applied magnetic field of $\mu_{0} \mathrm{H}=1 \mathrm{~T}$. The line 
represents the Debye heat capacity model. The inset figure is the $\mathrm{C}_{\mathrm{p}} / \mathrm{T}$ vs $\mathrm{T}$ plot showing more clearly the magnetic transition around $10 \mathrm{~K}$.

Figure 8: Magnetization vs H/T for $\mathrm{T}=15$ and $30 \mathrm{~K}$, and curves of the Langevin function. At 15 and $30 \mathrm{~K}$, there are two phases, i.e. the ordered magnetic clusters and the PM phase described with the Langevin function and the Brillouin function, respectively. The fits give 50 and $10 \%$ for the proportion of clusters into the PM phase, and 100 and $30 \mu_{\mathrm{B}}$ for the cluster moment at 15 and $30 \mathrm{~K}$, respectively. Inset figure shows the good fit at $270 \mathrm{~K}$ with the Brillouin function.

Figure 9: Magnetic phase diagram (H-T) of the $\mathrm{Nd}_{49} \mathrm{Al}_{13} \mathrm{Ni}_{38}$ amorphous alloy constructed from present results. PM, SPM, FM, CG and SG stand for paramagnetic, superparamagnetic, ferromagnetic, cluster-glass and spin-glass domain, respectively. The various lines represent the characteristic temperatures $\mathrm{T}_{\mathrm{d}}, \mathrm{T}_{\mathrm{C}}, \mathrm{T}_{\mathrm{irr}}, \mathrm{T}_{\mathrm{f}}$ which are summarized Table 1.

Figure 10: Temperature dependence of the magnetic entropy of the $\mathrm{Nd}_{49} \mathrm{Al}_{13} \mathrm{Ni}_{38}$ amorphous corresponding to $\mu_{0} \Delta \mathrm{H}=1 \mathrm{~T}$ and calculated from equation 2 . 
Figures

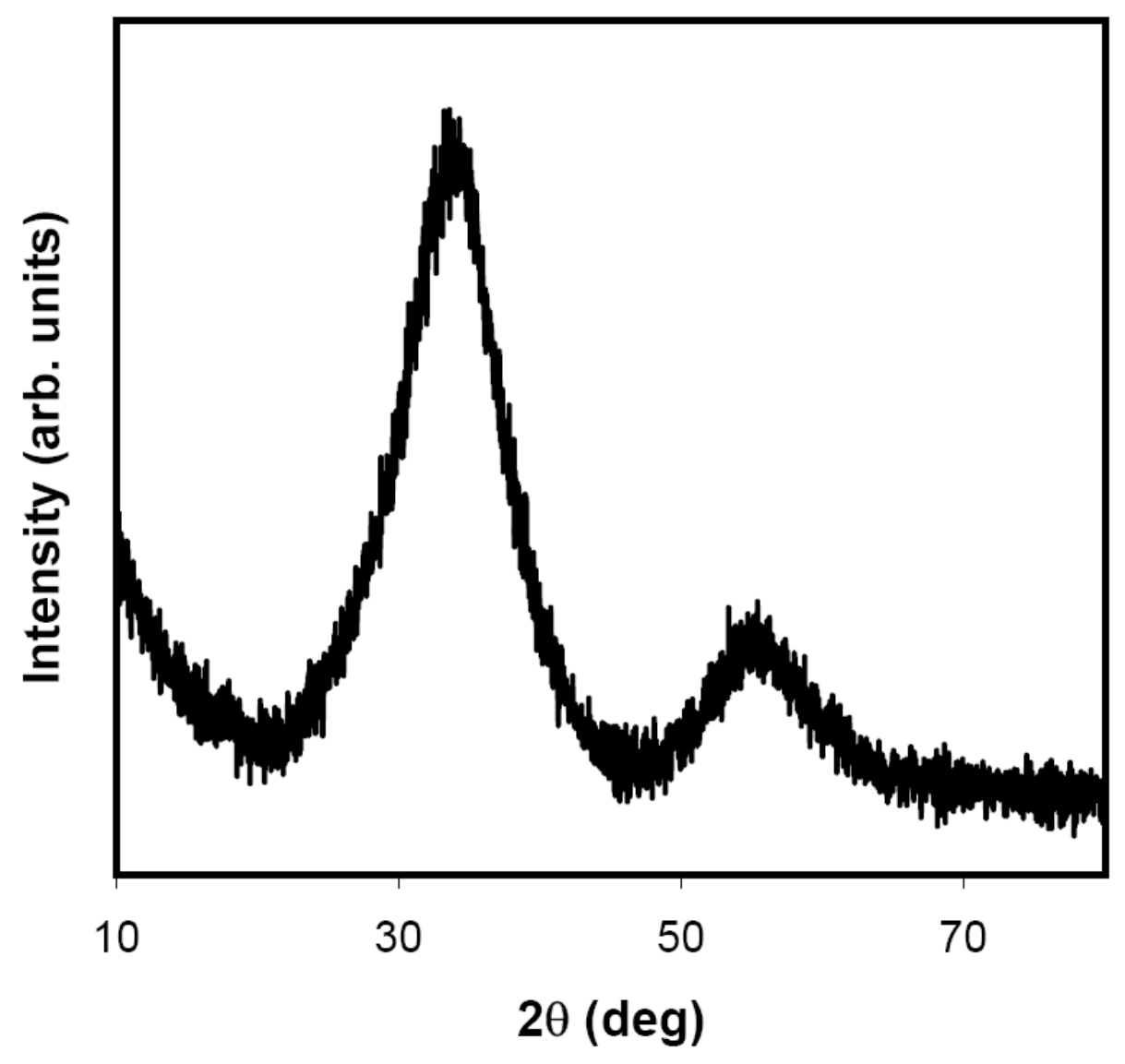

Figure 1: 


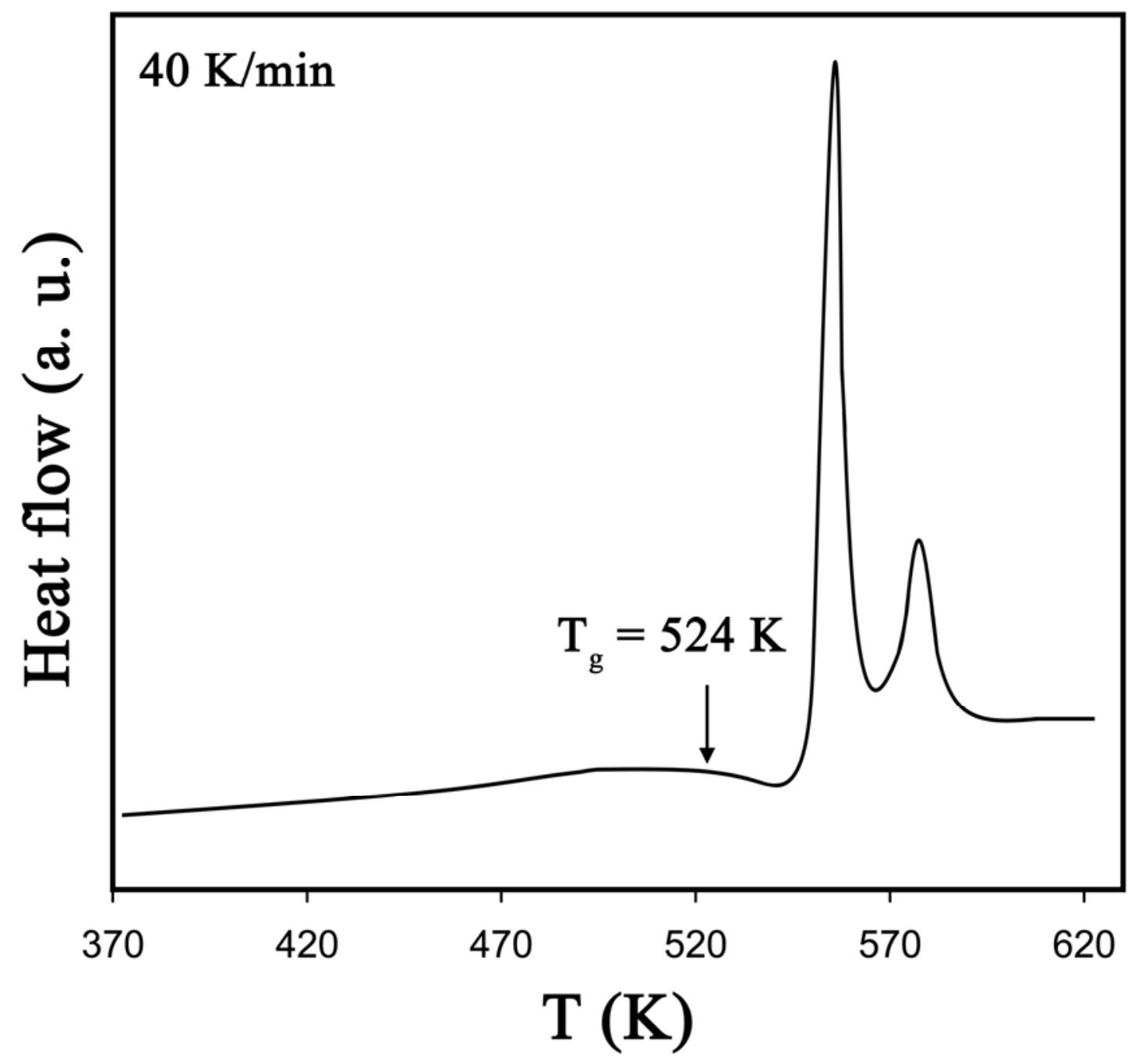

Figure 2: 


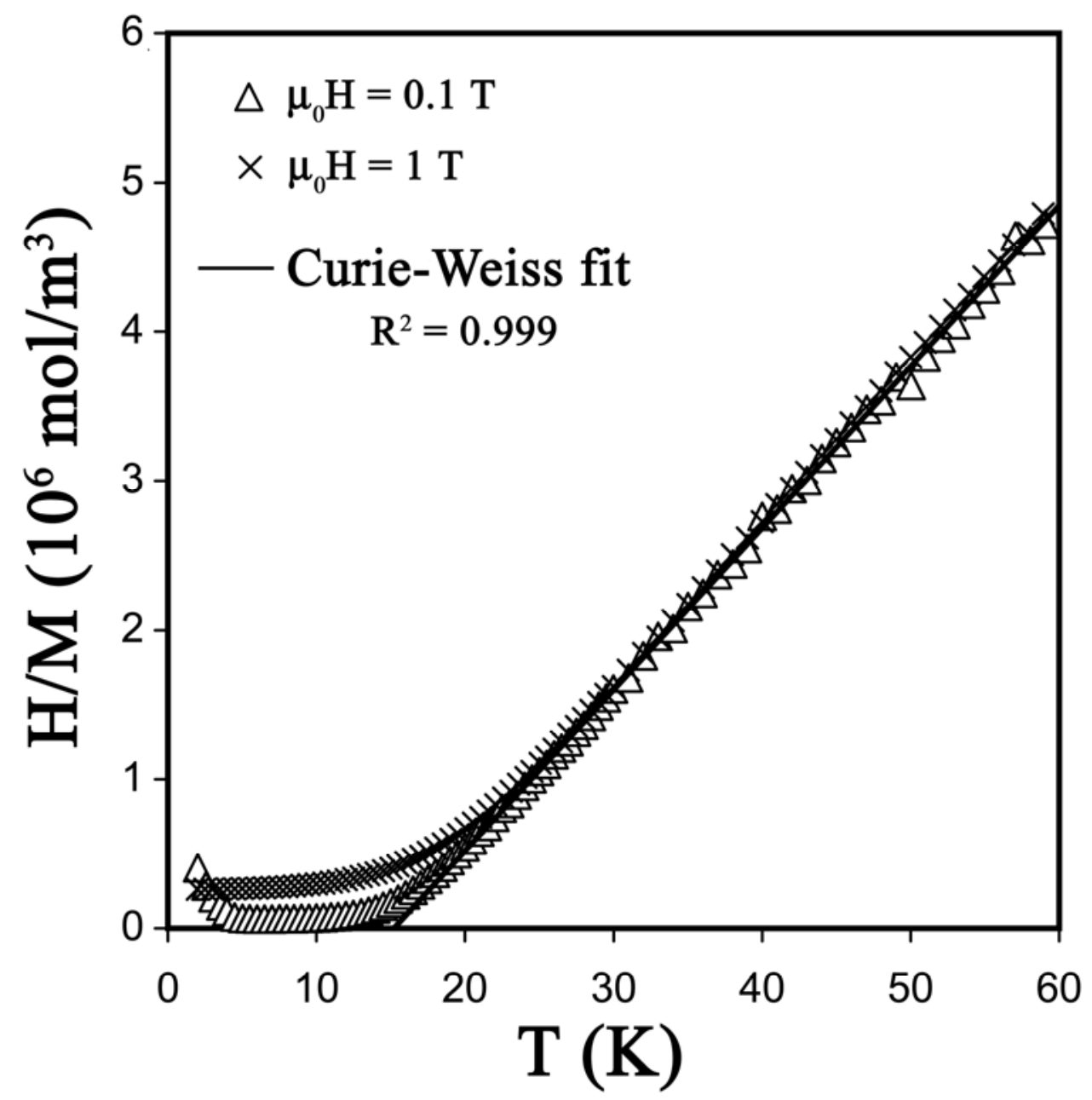

Figure 3: 


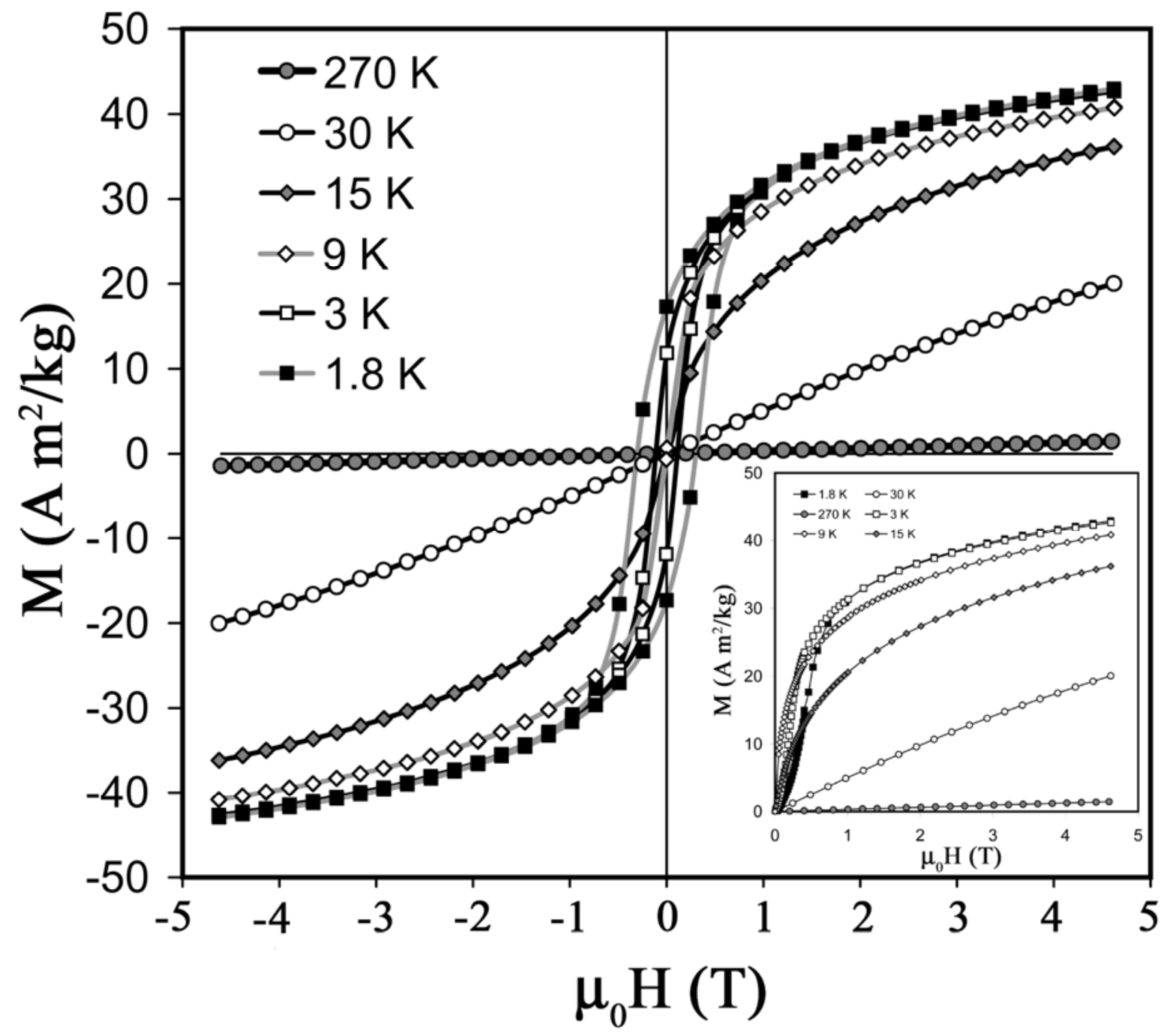

Figure 4 


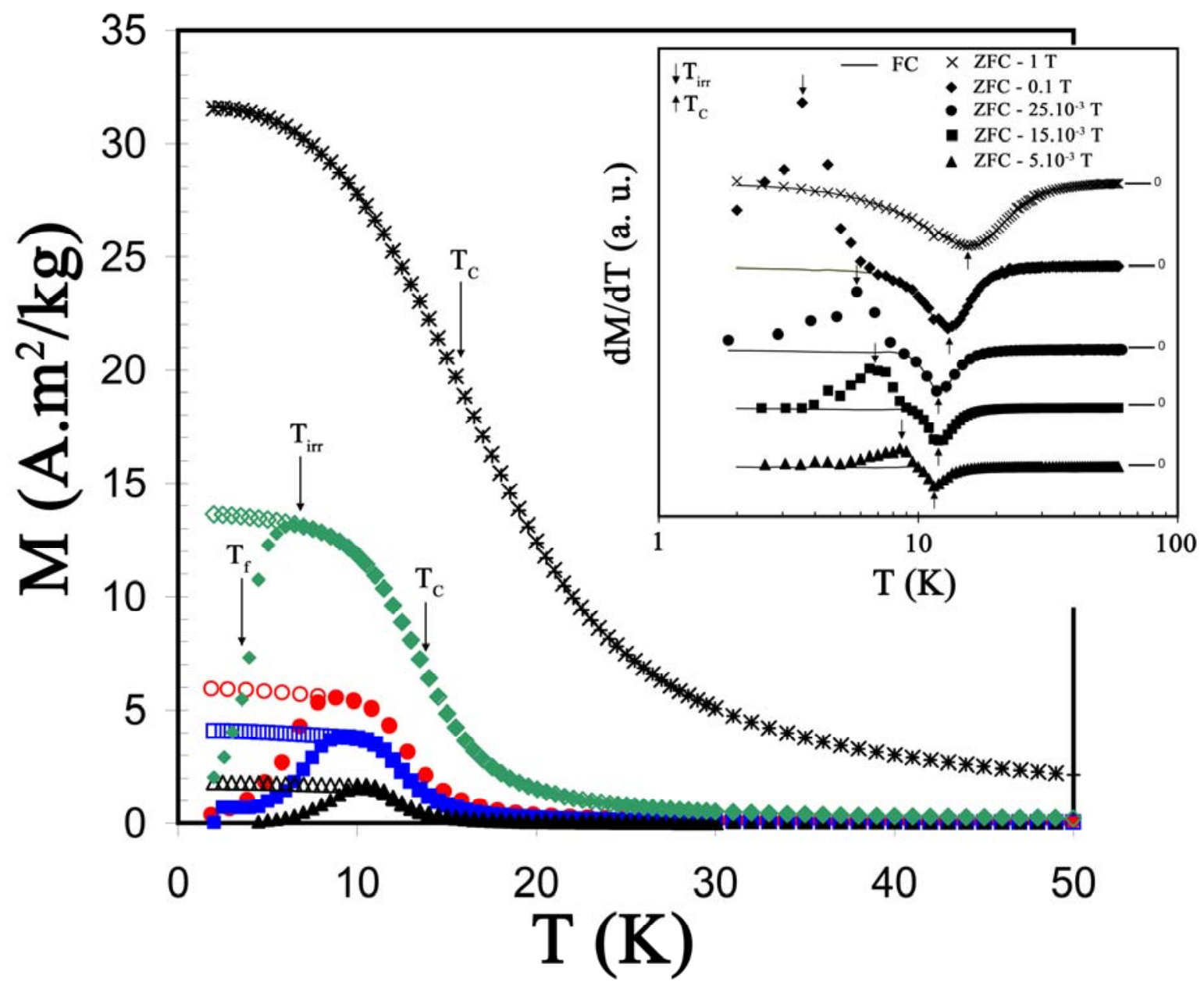

Figure 5: 


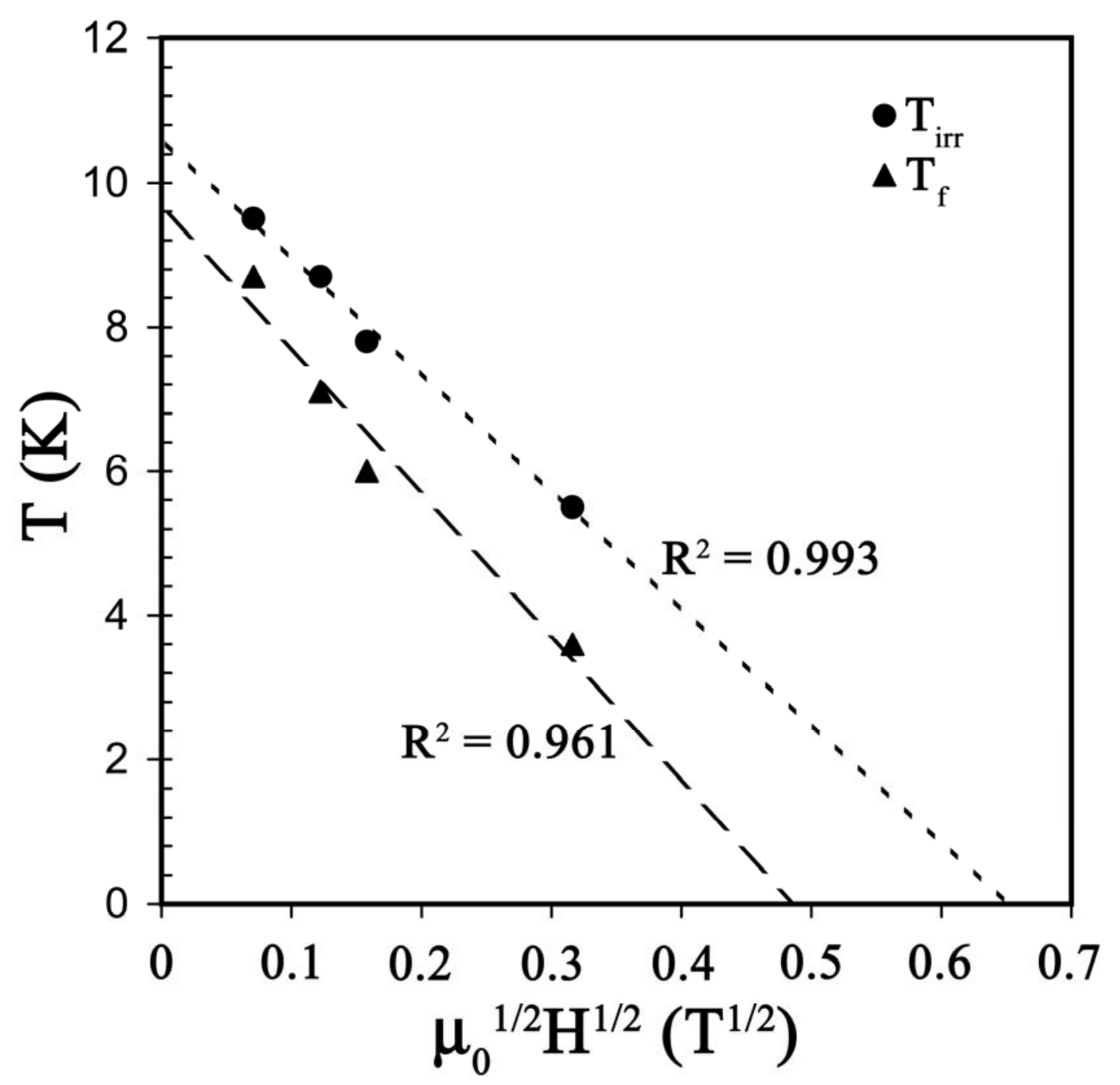

Figure 6 


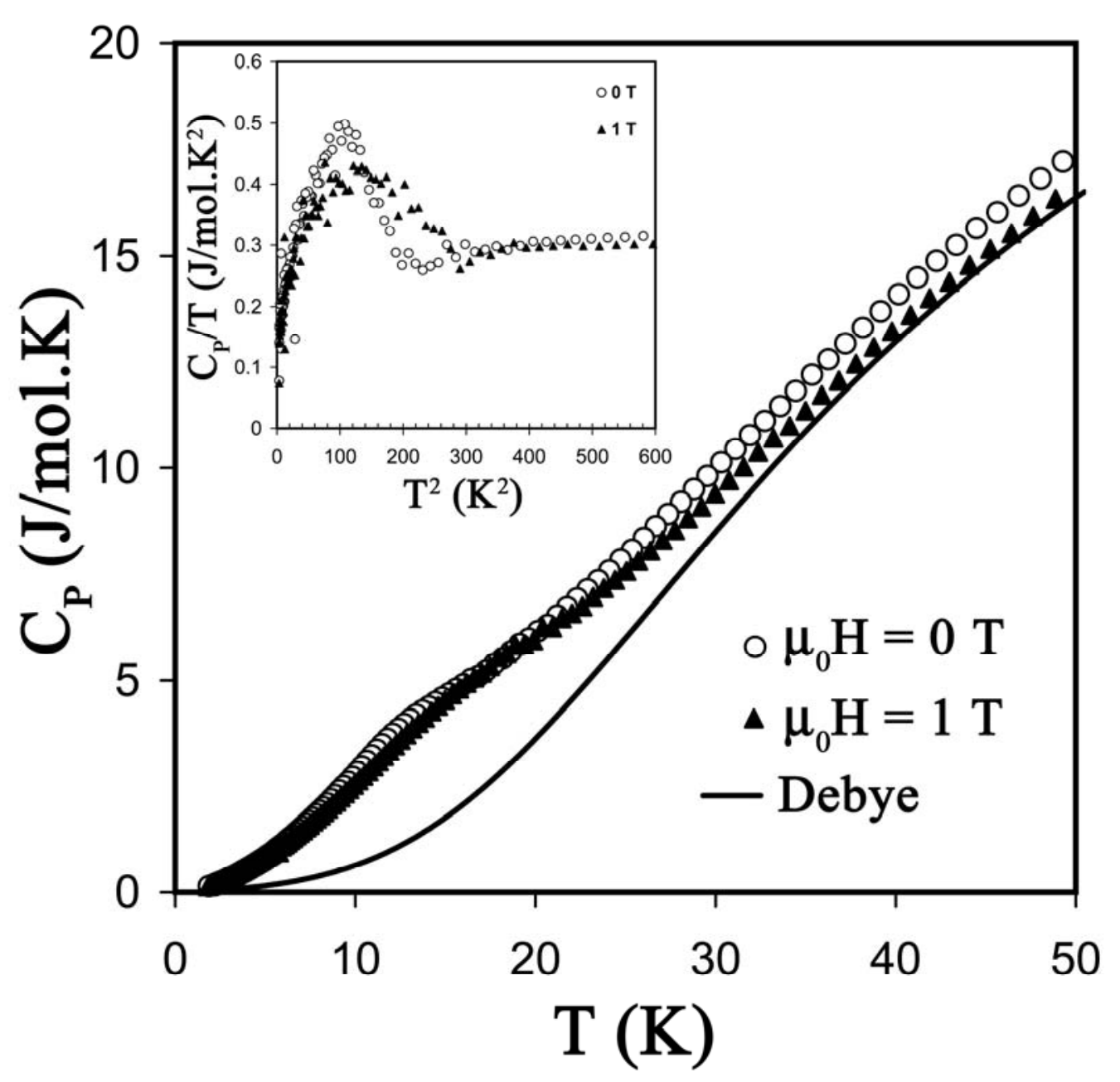

Figure 7 


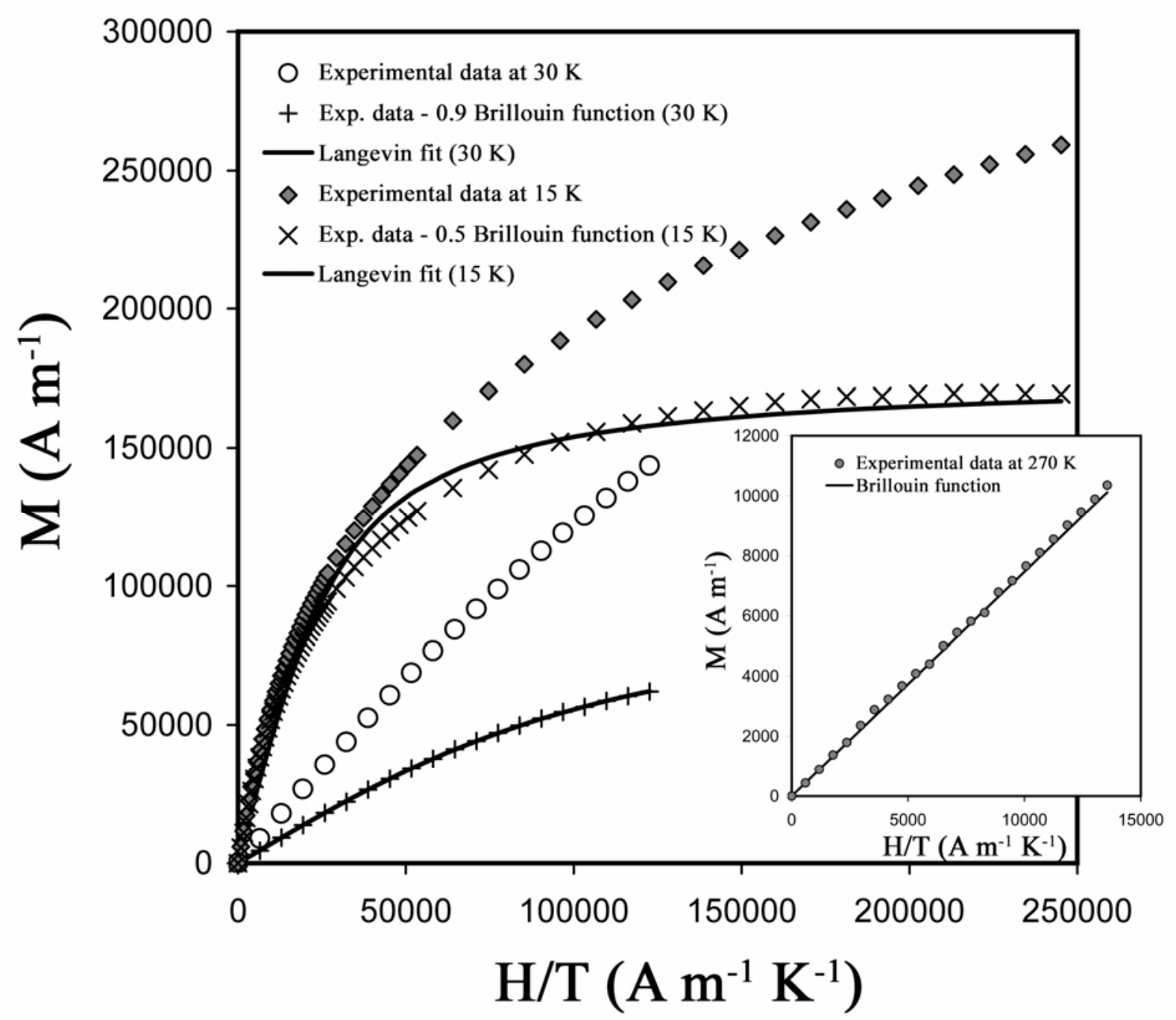

Figure 8 : 


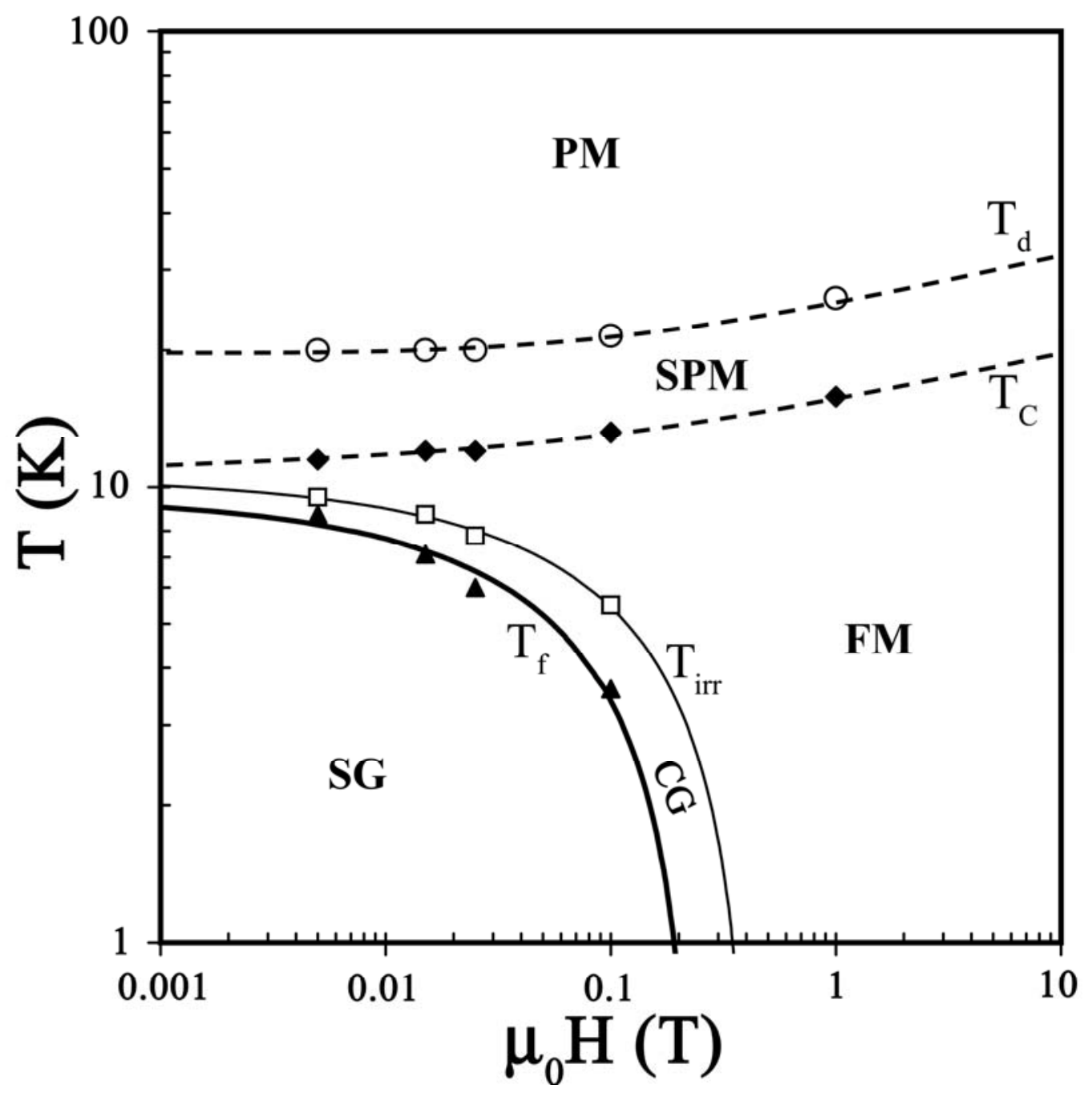

Figure 9 


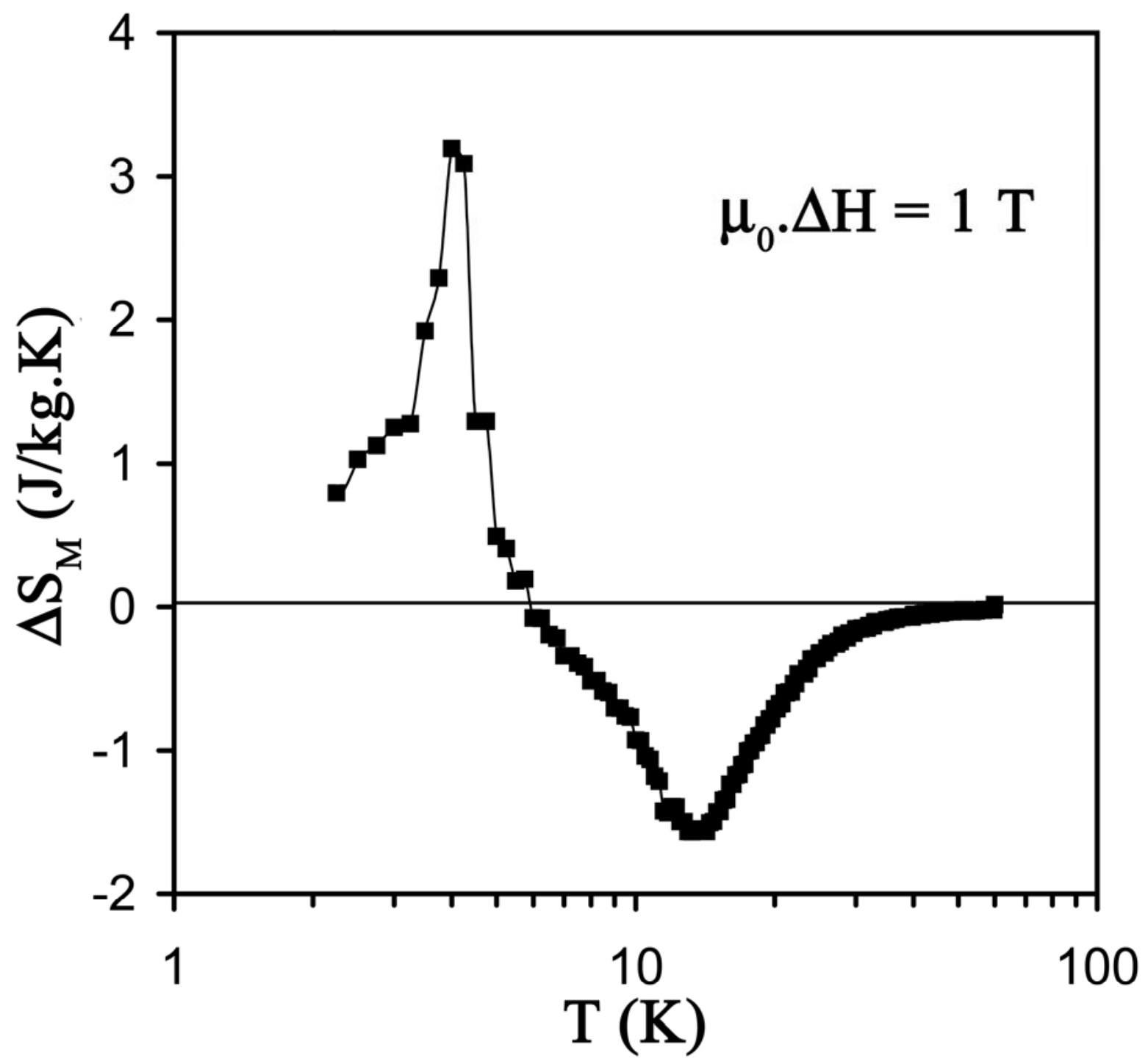

Figure 10 